\title{
Cenários futuros para a produção de bovinos de corte no Brasil
}

Submissão: 25/08/2021

Aceite: $10 / 10 / 2021$

\begin{abstract}
Resumo
O objetivo deste artigo é analisar o futuro da produção de bovinos de corte no Brasil em comparação com evidências já encontradas na literatura. Para atingir tal proposta, é utilizado o documento "O futuro da cadeia produtiva da carne bovina brasileira: uma visão para 2040" como base documental para a busca de indicativos de ocorrência destas tendências no país. Foi realizada uma análise documental na publicação técnica de visão para 2040 para o elo de produção da cadeia de produtiva e uma pesquisa em bases internacionais em busca de pesquisas com evidências empíricas no contexto brasileiro. Os resultados mostraram que é possível evidenciar que todos os temas gerais sobre o uso de terras, gestão, estrutura produtiva, manejo, meio ambiente e comercialização de animais, já foram abordados em pesquisas, assim como a maioria de seus temas específicos. Com exceção da orientação para tecnologia no campo, que ainda é um desafio para grande parte dos pecuaristas devido ao seu custo elevado de implementação. Todos os outros subtemas que foram correlacionados com as evidências, estão indo ao encontro das tendências apresentadas no documento sobre o futuro da produção brasileira de bovinos de corte. A grande relevância do documento da Embrapa é apresentar as tendências que oferecem aos tomadores de decisões, possíveis cenários para formulação de estratégias que o tornem mais competitivo no mercado de carne bovina.
\end{abstract}

Palavras-chave: Bovinocultura de corte. Produção. Tendências. Cenários.

\section{Future scenarios for the beef cattle production in Brazil}

\begin{abstract}
The purpose of this article is to analyze the future of beef cattle production in Brazil in comparison with evidence already found in the literature. To achieve this proposal, the document "The future of the Brazilian beef production chain: a vision for 2040" is used as a documentary basis for the search for indications of the occurrence of these trends in the country. A documentary analysis was carried out in the technical publication of Vision for 2040 for the production link in the production chain and a search on an international basis in search of research with empirical evidence in the Brazilian context. The results showed that it is possible to show that all general themes on land use, management, productive structure, management, environment and commercialization of animals, have already been addressed in research, as well as most of their specific themes. With the exception of the orientation towards technology in the field, which is still a challenge for most of the ranchers due to its high cost of implementation. All other sub-themes that were correlated with the evidence, are in line with the trends presented in the document on the future of Brazilian beef cattle production. The biggest relevance of the Embrapa document is to introduce the trends that offer decision-makers possible scenarios for formulating strategies that make them more competitive in the beef market.
\end{abstract}

Keywords: Beef cattle. Production. Tendencies. Scenarios.

\footnotetext{
${ }^{1}$ Doutoranda em Administração (PPGAD/UFMS). https://orcid.org/0000-0001-9485-4637. Email:santos.paulads@gmail.com

${ }^{2}$ Doutorando em Administração (PPGAD/UFMS). https://orcid.org/0000-0002-5140-1918. Email:weslei.maique@gmail.com

${ }^{3}$ Doutorado e Pós-Doutorado em Administração (UFMS). Professora do Programa de Pós-Graduação em Administração Pública (ESAN-UFMS). https://orcid.org/0000-0001-9363-9716.Email:yasmin.casagranda@ufms.br

${ }^{4}$ Doutorado em Agronegócios (UFRGS). Professor do Programa de Pós-Graduação em Administração (UFMS). Pesquisador da Embrapa Gado Corte (CNPGC). https://orcid.org/0000-0001-8565-1053

Email:guilherme.malafaia@embrapa.br
} 


\section{Introdução}

O Brasil é o maior exportador de carne bovina do mundo, sendo o segundo maior país no número de cabeças e de quantidade de carne produzida (USDA, 2021a). Este posicionamento coloca o país em posição de destaque diante do mercado, bem como mostra a capacidade de atendimento da sua produção aos mercados consumidores que têm demanda pelo produto. Os destinos de destaque destas exportações são China, Hong Kong, Egito e Chile, com destaque para as exportações do primeiro que foram quadriplicadas entre 2010 e 2020 (MDIC, 2021).

Dentro do país, os principais estados produtores são Santa Catarina, São Paulo, Rio Grande do Sul, Pernambuco e Mato Grosso do Sul. O conjunto de exportações destes estados compreende $96,8 \%$ de todas as exportações brasileiras, mostrando a alta representatividade destes (MDIC, 2021). Faz-se questão determinante o estudo dos seus processos produtivos para que esta relevância para o mercado mundial acompanhe as tendências previstas para o futuro do setor.

Os estudos sobre o contexto de desenvolvimento do agronegócio vêm acompanhados de discussões sobre a dilatação do uso de tecnologias e melhorias na cadeia de alimentos (AGROPENSA, 2014). Essa preocupação da ciência passou a ser acompanhada de propensão aos aprofundamentos dos estudos com o objetivo de entender o futuro do tema e quais os seus desafios (FAO, 2017).

Em conhecimento do contexto e dos avanços que estão sendo observados na tecnologia do setor, buscou-se determinar os desafios enfrentados pela produção de carne bovina para os próximos anos. Na literatura mais recente, a visão para 2040 foi descrita a partir da construção de cenários do setor e aponta as principais tendências para a produção no que tange o uso de terras, gestão, estrutura produtiva, manejo, meio ambiente e comercialização de animais (EMBRAPA, 2020).

Neste sentido, a análise de cenários sobre aspectos da produção com o apresentado em publicações científicas pode nortear pontos de atenção e melhoria para a prática. Dentre o que já foi apresentado pela ciência estão pesquisas quanto à adoção das tecnologias (GROHER; HEITKÄMPER; UMSTÄTTER, 2020) e smart farming (PIVOTO et al., 2018); dados para a análise de tendências (HU et al., 2019); novas abordagens e blockchain para segurança alimentar (HU et al., 2019); e percepções sobre o uso e integração dos sistemas produtivos (CORTNER et al., 2019).

Este artigo tem como objetivo analisar o futuro da produção de bovinos de corte no Brasil em comparação com evidências já encontradas na literatura. Para atingir tal proposta, é utilizado 
o documento da Embrapa (2020) "O futuro da cadeia produtiva da carne bovina brasileira: uma visão para 2040" como base documental para a busca de indicativos de tal ocorrência de tais tendências no país.

\section{Revisão de literatura}

\subsection{Produção de bovinos de corte no Brasil}

A crescente demanda por alimentos derivados da pecuária bovina é de nível global, devido aos ganhos de propriedades nutricionais que promovem a melhoria da segurança alimentar. Assim sendo, fomenta discussões cada vez mais recorrentes sobre a expansão da produção e o manejo dos recursos naturais (ENAHORO et al., 2019).

O setor produtivo de carne bovina brasileiro, tende a ser enaltecido e valorizado cada vez mais, nacionalmente pela sua importância no país e, globalmente devido as exportações (EMBRAPA, 2020). A produção global de carne está estimada $2 \%$ ano a ano, e tem-se a expectativa de que a produção do Brasil seja maior pela demanda doméstica e a exportação, sobretudo para China, devido à queda da produção de suínos (USDA, 2021b).

A produção de bovinos de corte está inserida na rede complexa da sua cadeia. Entre os elos é possível identificar desde o processo produtivo, até o seu processamento, logística, armazenagem e varejo/comércio de alimentos (CICARNE, 2021). Ressalta-se a relação entre todos os agentes envolvidos na figura 1.

Figura 1 - Cadeia produtiva da bovinocultura de corte.

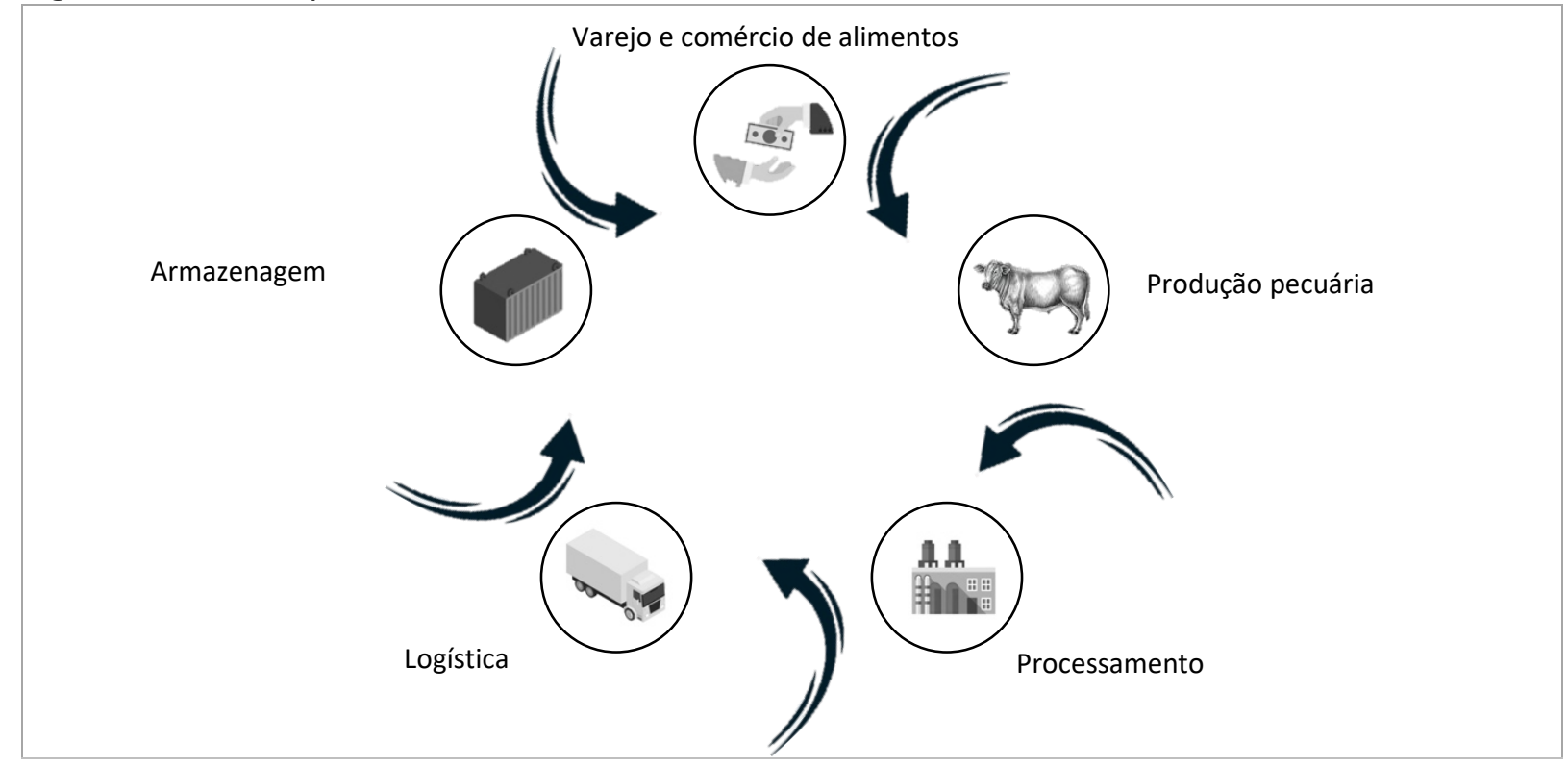

Fonte: Dados da pesquisa. 
Na produção pecuária pauta-se o objetivo desta pesquisa. O Brasil possui três sistemas de produção de bovinos predominantes: extensivo, semi-intensivo e intensivo (CEZAR et al., 2005). Compreende-se sistema de produção da pecuária de corte como sendo um conjunto de tecnologias e práticas de manejo e que contém diferentes combinações de composição do ciclo de produção: cria, recria e engorda. (MALAFAIA et al., 2019).

Caracteriza-se os sistemas extensivos pela utilização de pastagens nativas e cultivadas como única fonte de alimentos energéticos, ou seja, sendo regime exclusivo de pastagem (CEZAR et al., 2005). Devido as suas características, esse sistema de produção, pode colocar em risco o desempenho geral da pecuária (DICK; SILVA; DEWES, 2015).

Enquanto o sistema semi-intensivo utiliza-se de pastagem e suplementação em pasto e, o intensivo lida com pastagem, suplementação e confinamento. (CEZAR et al., 2005). As práticas no sistema intensivo da pecuária bovina é tida como mais eficiente e que causam menos impactos ambientais comparado ao sistema extensivo (SOBROSA NETO et al., 2018).

Posto isso, nota-se a diversidade dos modelos de produção de carne bovina atrelados a demandas globais sustentáveis de dimensões sociais, ambientais, econômicas e institucionais. Para manter a liderança no mercado global, a gestão da cadeia de carne precisa estar aliada a ferramentas eficientes, tais como a análise de cenários futuros para fazer frente aos desafios postulados. (EMBRAPA, 2020; CEZIMBRA et al., 2021).

\subsection{Cenários futuros como estratégia para o setor produtivo}

Dadas as implicações da economia globalizada, a velocidade das mudanças advindas da tecnologia, promovendo mudanças de comportamento de produção e de consumo da sociedade e a escassez de recursos naturais, o campo de incertezas vem crescendo constantemente. A fim de assegurar-se das melhores ações homem-ambiente (THEISSEN et al., 2019) diante de tantas mudanças, as técnicas de cenários auxiliam os tomadores de decisões a lidarem com o que está acontecendo no presente e possíveis tendências para o futuro.

Esses desafios de mercado, enaltecem estudos de cenários pois lidam com questões de várias escalas, desde vínculos institucionais, mudanças no uso das terras, tecnologias, questões socioeconômicas e até mesmo políticas de desenvolvimento de âmbito nacionais e internacionais (DÍAZ et al., 2015). A construção de cenários permite diferentes perspectivas entre os níveis de desenvolvimento. Sendo uma ferramenta que norteia as partes envolvidas em 
subsistemas sociais, econômicos e ambientais em interação mútua, complexas e rodeadas de incertezas intrínsecas à gestão (OTEROS-ROZAS et al., 2015; PEARSON et al., 2017).

O desenvolvimento de cenários sobre os modelos de produção podem fornecer suportes para políticas públicas e, também, esclarecer a relação entre as mudanças no uso da terra, tecnologias aplicadas nos processos e nos serviços de ecossistêmicos (XU et al., 2018). Ademais, o planejamento de cenários futuros permite a exploração e antecipação de incertezas de temáticas complexas (OUKO et al., 2020) , tal como a produção de carne bovina.

A produção da pecuária bovina sofreu evolução em seus sistemas nas últimas décadas, devido a competição entre os produtores e a alta demanda global, neste sentindo, faz-se necessário cada vez mais análises de cenários futuros que detalhe possíveis mudanças estruturais e não estruturais dos sistemas de produção, a fim de manter a gestão eficiente (NISKANEN; IHO; KALLIOVIRTA, 2020).

\section{0 método de pesquisa}

Nesta pesquisa são utilizados os métodos de revisão bibliográfica e análise de conteúdo em sua vertente de análise documental, que é uma maneira de representar o conteúdo de um documento de uma forma diferente da original, com o objetivo de facilitar a consulta e referenciação posterior do mesmo (BARDIN, 2016). Para tal, é seguida a técnica proposta por Bardin (2016) composta por três etapas: a (1) a pré-análise; (2) a exploração do material e (3) o tratamento dos resultados, a inferência e interpretação.

Na primeira etapa, a pré-análise, existem três fatores a serem contempladas: (1) a escolha do documento; (2) formulação de hipóteses; e (3) definir objetivos para a interpretação final. Os três fatores não precisam, necessariamente, seguir uma ordem, pois estão ligados uns aos outros em uma relação de interdependência (BARDIN, 2016). Nesta pesquisa, o documento "O futuro da cadeia produtiva da carne bovina brasileira: uma visão para 2040" foi escolhido como fonte central de dados secundários, com o objetivo realizar uma comparação com evidências empíricas em publicações cientificas que possam ser observadas as tendências que já estão em curso (EMBRAPA, 2020).

Por se tratar de um documento de tendências com base em um estudo utilizando a técnica Delphi, as hipóteses estabelecidas serão de ordem afirmativa, ou seja, as tendências estabelecidas no documento serão consideradas hipóteses positivas em relação ao que é evidenciado empiricamente. As tendências são divididas entre os elos da cadeia de produção de 
carne bovina, porém, para que seja possível uma análise mais aprofundada, este estudo foi limitado às tendências ao elo produtivo apresentadas na quadro 1.

Quadro 1 - Tendências para a produção de carne bovina.

\begin{tabular}{|c|c|c|}
\hline \multicolumn{2}{|r|}{ Tendência em 2040} & Cod. \\
\hline \multirow{3}{*}{ Uso de Terras } & Integração pecuária de corte é fator relevante para expansão agrícola & UT1 \\
\hline & Diminuição da área de pastagem traz mais rentabilidade ao produtor & UT2 \\
\hline & Demarcação de terras indígenas na mão da opinião pública & UT3 \\
\hline \multirow{8}{*}{ Gestão } & As fazendas se tornando empresas por oportunidade e necessidade & GT1 \\
\hline & A continuidade do crescimento da pecuária necessita de melhor gestão & GT2 \\
\hline & Pecuarista mais jovem traz mais técnica à produção bovina & GT3 \\
\hline & A gestão na ponta dos dedos & GT4 \\
\hline & Orientação leva tecnologia ao campo & GT5 \\
\hline & Apagão de mão-de-obra & GT6 \\
\hline & Especialização para mão de obra permanecer no campo & GT7 \\
\hline & Políticas públicas em prol da pecuária são necessárias, mas pouco previsíveis & GT8 \\
\hline \multirow{7}{*}{$\begin{array}{l}\text { Estrutura } \\
\text { Produtiva }\end{array}$} & Êxodo rural diminui o número de pecuaristas de corte & EP1 \\
\hline & Centro-oeste pode perder o posto de maior produtor de bovinos de corte do Brasil & EP2 \\
\hline & Taurinos e Zebuínos disputam os holofotes do pecuarista & EP3 \\
\hline & ILPF em franca expansão & EP4 \\
\hline & Semiconfinamento equilibra produtividade e bem-estar animal & EP5 \\
\hline & Uso de confinamento segue imprevisível & EP6 \\
\hline & $\begin{array}{l}\text { Confinamento terceirizado facilita a terminação de bovinos de corte, mas ainda com } \\
\text { entraves }\end{array}$ & EP7 \\
\hline \multirow{7}{*}{ Manejo } & Técnicas de manejo bem estabelecidas sendo disseminadas & MJ1 \\
\hline & Tecnologia aliada à necessidade de melhorias no gerenciamento & MJ2 \\
\hline & $\begin{array}{l}\text { Imposições externas de boas práticas de manejo forçam a sua disseminação no } \\
\text { Brasil }\end{array}$ & MJ3 \\
\hline & Produtos de reuso são encontrados em instalações pecuárias & MJ4 \\
\hline & Suplementação proteica via consócio ou via cocho & MJ5 \\
\hline & A tecnificação traz avanços às pastagens & MJ6 \\
\hline & Recuperar pastagens degradadas é quase uma obrigação & MJ7 \\
\hline \multirow{4}{*}{$\begin{array}{l}\text { Meio } \\
\text { Ambiente }\end{array}$} & Geração da própria energia cresce no Brasil mesmo com certos entraves & MA1 \\
\hline & O aumento na produtividade auxilia o meio ambiente & MA2 \\
\hline & Redução do consumo de água pela pecuária & MA3 \\
\hline & A priorização das questões ambientais no Brasil & MA4 \\
\hline \multirow{3}{*}{$\begin{array}{l}\text { Comercializaçã } \\
\text { o de Animais }\end{array}$} & Especialização do comércio de animais é sinal de evolução no sistema produtivo & CA1 \\
\hline & Questionamento para o bem-estar animal impactam negociações comerciais & CA2 \\
\hline & A modernização dos leilões no Brasil & CA3 \\
\hline
\end{tabular}

Fonte: Adaptado de EMBRAPA (2020).

$\mathrm{Na}$ etapa de exploração do material são aplicadas as decisões estabelecidas na primeira etapa. Primeiramente, é apresentada uma descrição analítica de todas as tendências para o elo de produção de carne bovina, destacando suas conclusões centrais que, posteriormente, são confrontadas com evidências empíricas. Foram selecionadas as bases Web of Science, Scopus e 
Science Direct para a busca de evidências empíricas entre 2016 até a data de coleta dos dados em março de 2021.

Para a busca, foram utilizadas as tendências como palavras-chave em conjunto com pecuária de corte ("beef cattle"): Uso de terras ("Iand use"); Gestão ("management"); Estrutura produtiva ("productive structure"); Manejo ("production systems" or "livestock production"); Meio ambiente (environment); e Comercialização de animais ("commercialization"). Para revisão bibliográfica foram definidos dois critérios de seleção dos artigos: primeiro, o estudo ter analisado o contexto brasileiro; e segundo: que os resultados se relacionassem com as tendências apresentadas no documento de cenários para 2040.

No tratamento dos resultados, a inferência e interpretação, é apresentado um quadro comparativo. Os artigos foram avaliados e comparados com as tendências com o objetivo de identificar uma relação positiva (+) ou negativa (-), possibilitando a evidenciação de tendencias que já estão em curso e se estão seguindo a direção indicada por especialistas na metodologia Delphi utilizada no documento de visão para 2040 para pecuária brasileira.

Na sequência é dada uma breve descrição do documento utilizado desse estudo, seguindo para a apresentação das tendências para uso de terras, gestão, estrutura produtiva, manejo, meio ambiente, comercialização de animais e suas respectivas comparações com as evidências empíricas.

\section{Resultados e discussão}

O documento selecionado para esta análise é intitulado "O futuro da cadeia produtiva da carne bovina brasileira: uma visão para 2040", um projeto desenvolvido em conjunto pelo Centro de Inteligência da Carne Bovina (CiCarne), a Embrapa Gado de Corte e o Ministério da Agricultura, Pecuária e Abastecimento (MAPA). O objetivo do documento é descrever possíveis cenários para cada elo de produção de carne bovina, ou seja, os elos de insumos, produção de bovinos de corte, indústria frigorífica, comercialização e consumo (EMBRAPA, 2020).

Utilizando a metodologia Delphi, foi obtido um prognóstico estruturado sobre os desafios tecnológicos para a cadeia de pecuária de corte no Brasil para 2040, dando origem aos cenários com o tema "Desafios para a pecuária de corte no Brasil para 2040" (EMBRAPA, 2020). Os cenários são construídos a partir da probabilidade de ocorrência de alguns eventos que podem influenciar o futuro, estes eventos são chamados de eventos possíveis, e combinando a 
probabilidade dos eventos possíveis, é possível estimar a probabilidade de concretização de um ou mais cenários (EMBRAPA, 2020).

Os eventos e suas probabilidades foram construídos a partir da opinião de especialistas combinadas, coletadas em tapas sucessivas. Apesar disso, os desafios para pecuária são amplos e possui fatores interdependentes, portanto, para complementar o estudo, foi realizada uma análise de impactos cruzados, procurando a influência de eventos entre si, por meio do cálculo de probabilidades condicionais, tendo como base o teorema de Bayes e simulação de Monte Carlo, com o auxílio de software específico (EMBRAPA, 2020).

\subsection{Uso de terras}

O uso das terras para pastagens começou a concorrer com as áreas destinadas às lavouras nos últimos anos. Os dados apontam uma queda de $5 \%$ nas áreas de pastagens e um aumento de 6\% nas áreas de lavoura (IBGE, 2017). Os estudos apontam que a tendência para 2040 é que as áreas destinadas para lavouras seja a mesma destinada às pastagens, visto que atualmente a área de pastagens é 2,4 vezes maior que a área destinada à agricultura.

Está tendência é corroborada pela tecnificação da pecuária e expansão da agricultura brasileira, que, em conjunto aos cuidados nutricionais, sanitários e manejo proporcionarão um maior aproveitamento das terras. Os resultados desse processo apontam para um encorajamento da agricultura orgânica e reforma das terras (VILPOUX; GONZAGA; PEREIRA, 2021); preocupação com o valor socioeconômico local (NUNES et al., 2019); e estes impactos no comércio mundial (ZU ERMGASSEN et al., 2020). Estes fatores contribuirão para a redução de áreas de pastagens em comparação aos dias atuais. Onde há sistemas de produção com maior tecnologia, por exemplo, há uma tendência de aumento de produtividade mesmo com uma redução de área (EMBRAPA, 2020).

Mesmo com um aumento da produção de carne bovina em quatro vezes nos últimos anos 40 anos (EMBRAPA, 2021), com base no Cadastro Ambiental Rural - CAR, o Brasil possui 66,3\% da sua área em preservação e proteção de vegetação nativa e biodiversidade (EMBRAPA TERRITORIAL, 2020). Muitas dessas áreas são terras indígenas, terras devolutas, assentamentos, militares e outras categorias socioambientais. A tendência é que haja acordos de parcerias entre produtores e responsáveis por estas áreas, principalmente das terras indígenas. Estas ações dependem de ações políticas, normas do estado e ainda é incerto como será decorrer destas negociações, mas são esperados diversos entraves para essa decisão (EMBRAPA, 2020). 


\subsection{Gestão}

Apesar de pesquisas apontaram que a capacidade de gestão e de organização dos pecuaristas atuais se resume em anotações em papéis desconexos e com a maioria não realizando nenhum tipo de controle, a tendência para 2040 é que o pecuarista tome posição de empresário e veja sua propriedade como uma empresa (EMBRAPA, 2020). Adoção de processos de gestão e melhorias no aumento de produtividade por meio de uso de tecnologias, dentro das tendências de sustentabilidade e bem-estar animal, são práticas que se tornarão fundamentais neste processo de mudança (LOPES et al., 2018; STABILE et al., 2020).

Outro fator que forçará uma melhor gestão da pecuária é o seu próprio crescimento. A pecuária está em constante expansão e passa demandar soluções de infraestrutura logística, investimento em $P \& D$, redução de custos, controle de doenças e aumento na eficiência no uso dos recursos naturais (EMBRAPA, 2020). Todos estes fatores demandam um alto nível gerencial, mas uma grande barreira é o desinteresse dos pecuaristas em se adaptar, querendo manter os antigos costumes na lida da fazenda, podendo desacelerar o processo de tecnificação da gestão.

A redução da idade média dos pecuaristas pode ser um diferencial nesse processo de mudança. Apesar da sucessão rural no país ainda ser um problema, em que há desinteresse dos filhos de proprietários em dar continuidade aos negócios da família, existem investidores nos sistemas pecuários que estão buscando melhorias gerenciais e profissionalização no setor (BARBOSA et al., 2020). Os jovens têm maior acesso ao estudo, isso faz com que sucessores e novos investidores tenham mentalidade diferente, contando com conhecimentos técnicos pecuários e agrícolas, sendo mais receptivos a tecnologias de precisão de produtividade (MENESES; GOMES, 2020).

Aplicativos de gestão e controle da propriedade serão comuns, sofrendo grande influência dos pecuaristas mais jovens, o uso de tecnologias e sua disseminação possibilita diminuição de custos, deixando sistemas como este mais atrativos financeiramente. Para os pecuaristas tradicionais, vai ser necessário treinamento para alcançar essa adaptação, assim como para a mão-de-obra na tentativa de evitar o apagão (EMBRAPA, 2020).

Para receber essas mudanças tecnológicas, é esperado que a maioria dos pecuaristas estejam recebendo orientação técnica baseada em pecuária de precisão. Esta tendência vai contra o que é observado atualmente, estima-se que apenas $20 \%$ das propriedades rurais recebam orientação técnica de diferentes meios, sejam eles privados ou públicos (IBGE, 2019; STABILE et al., 2020). Um fator que pode inibir os pecuaristas são os custos da infraestrutura 
muito elevados, porém há uma tendência de diminuição do investimento necessário, tornandoa mais acessível (LOPES et al., 2018).

O êxodo rural gera uma alta rotatividade de mão-de-obra no campo, isso faz com que produtores se sintam desestimulados a oferecer treinamentos. Porém, a introdução de máquinas no dia a dia do campo tem trazido mudanças nas relações de trabalho, tornando necessária a maior qualificação de mão-de-obra e diminuindo o uso da mesma em comparação há alguns anos. Durante o primeiro ano da pandemia de SARS-CoV-2, em 2020, houve uma redução de 5,2\% da população ocupada no agronegócio, agravando ainda mais a o cenário de apagão de mão-de-obra (CEPEA, 2021). No entanto, esta queda ainda é menor que o número de ocupados no Brasil (7,9\%), fazendo com que a participação do agronegócio no mercado de trabalho aumentasse 0,6\% em relação à 2019 (CEPEA, 2021).

Busca-se entendimento de como será a mão de obra no campo. A tendência é que cada vez mais necessita-se mão de obra qualificada, o que se tem pouco na realidade pecuária. Isso é causado pela falta de capacidade de liderança coerente e treinamentos, com isso é notável a importância de uma mudança no perfil gerencial para retenção de mão de obra de boa qualidade (CEBALLOS et al., 2018). Apesar de mais qualificada, a falta de liderança, pode levar estas pessoas a deixarem o campo.

Já foi mostrado que a falta a de mão de obra qualificada é e continuará sendo um dos maiores desafios na bovinocultura de corte. Com a alta rotatividade de mão-de-obra no campo faz com que os produtores sejam desestimulados a oferecer treinamentos. Em suas concepções, qualquer mudança no trabalho é sinônimo de abandono do cargo, outro fato é o não comprometimento por parte dos funcionários com os serviços.

As políticas de incentivo serão de grande importância para o desenvolvimento rural, temse visto maiores aportes financeiros à área rural no país, com maiores valores disponíveis e auxílio em pequenos produtores que tem maiores condições. Associações de entidades de desenvolvimento governamentais terão papel fundamental na melhora de relações comerciais na pecuária (EMBRAPA, 2020).

\subsection{Estrutura produtiva}

No Brasil, houve uma saída de mais de 1,5 milhões de trabalhadores do ramo agropecuário (IBGE, 2017). Projeções indicam que até 2040 grande parte dos pecuaristas de corte saia da atividade (EMBRAPA, 2020). Isso se deve ao maior êxodo rural e também pela migração 
dos pecuaristas para o ramo agrícola; migração esta, estimulada pela expansão territorial da agricultura com seus ciclos curtos e giro mais rápido de capital. A produtividade dos pecuaristas, junto aos rebanhos criados com média ou baixa tecnologia enfrentam muitos desafios, que impactam nas relações de troca.(BARBOSA et al., 2020)

O estado Mato Grosso se mantém em 1ㅇ lugar no ranking de maior produtor de bovinos de corte no Brasil, desde 2014. Entretanto, das 10 cidades com maior produção de bovinos, 4 dela são do Pará e somente 1 do Mato Grosso (ABIEC, 2019). A adoção de técnicas de integração lavoura-pecuária-floresta tem favorecido os estados da conjunção MAPITOBA na criação de bovinos de corte, podendo futuramente superar Mato Grosso.

Em relação as estruturas produtivas brasileiras, os sistemas de Integração Lavoura Pecuária Floresta resultam em maior sustentabilidade e lucratividade, sob esta perspectiva, aponta-se alta probabilidade destes sistemas aumentarem mais do que o dobro de sua área atual até 2040. Para que isso ocorra é necessário que haja cada vez mais transferência de conhecimento aos pecuaristas, evidenciando que é possível aliar a eficiência produtiva e econômica, potencializando o sistema lucrativo.

Os produtores estão aderindo ao semiconfinamento que equilibra a produtividade e bemestar animal, ao invés da principal técnica utilizada na terminação dos animais, a engorda em pasto(OLIVEIRA et al., 2020). Devido a heterogeneidade do setor, a disponibilidade de insumos e custos destes insumos, a adoção de semiconfinamento passa a ser influenciada menos positivamente. Contudo, outros benefícios dos sistemas de integração que deve ser explorado, o crescimento destes sistemas favorece o aumento da disponibilidade de insumos, os quais amenizam os custos do semiconfinamento e atrai a lucratividade que a sustentabilidade gera (EMBRAPA, 2020).

Ainda assim, o uso de confinamento segue imprevisível, pois, mesmo diante dos benefícios, a tradição do pecuarista em produzir gado a pasto pode diminuir o interesse pela técnica, principalmente se este não estiver associado a nenhuma prática de remuneração variável por produtividade e qualidade junto à empresa frigorifica.

O estudo da Embrapa (2020) aponta que o confinamento terceirizado é outra alternativa para os pecuaristas, apesar de o mercado ser instável, a adoção da terceirização na terminação de bovinos de corte tem vantagens em aumento da rotatividade, assim como o confinamento tradicional, com acréscimo de não necessitar de investimentos em infraestrutura. Em contrapartida ao confinamento, outras técnicas estão sendo mais divulgadas para terminação 
com mais eficiência a campo, atraindo assim para que o produtor mantenha seu estilo de produção adotando melhorias.

\subsection{Manejo}

A conscientização dos pecuaristas está sendo disseminada cada vez mais atualmente, devido à forte pressão do mercado demandando práticas produtivas cada vez mais adequadas às exigências do consumidor. Para atender a tais expectativas são necessárias melhorias contínuas de manejo de criação de bovinos: implementação de bem-estar animal; adequação de estruturas da fazenda; treinamento de trabalhadores; nutrição adequada; logística de transporte dos animais segura, tanto para o abate quanto para a revenda (EMBRAPA, 2020). A tendência é de que a tecnologia possibilite aos pecuaristas mais acesso a dados que favorecem a influência no setor de gado de corte, sendo inclusive, aliadas a probabilidade de mensurações aos registros dos parâmetros de monitoramento dos índices zootécnicos (LAMPERT et al., 2020).

As exigências de boas práticas implicarão diretamente no manejo de instalações para o manejo dos animais, tendo em vista que a pressão dos consumidores estão cada vez mais pautadas na sustentabilidade(AIKEN et al., 2020). Mesmo que grande parte destas pressões esteja sendo atendidas, cabe considerar que questões edafoclimáticas e diferenças culturais ainda são barreiras para a padronização do manejo pecuário. Por outro lado, de forma particular e independente das ações federais, os frigoríficos particulares têm bonificado a produção mais sustentável(PALHARES; MORELLI; NOVELLI, 2021).

Devido à extensão territorial das pastagens brasileiras, o manejo na utilização de banco de proteínas atualmente tem uma baixa taxa de aceitação pelo produtor de bovinos de corte. Como alternativa que vem sendo disseminada o consorcio de pastagens com cultivares resistentes de plantas leguminosas, que favorece o manejo e diminui a necessidade de suplementação no cocho, resultando em maior rentabilidade ao sistema produtivo (EBLING et al., 2021).

O setor pecuário ainda precisa de manutenções na produtividade, tais como medidas para maior conscientização dos pecuaristas com os cuidados em âmbito sustentável para com as pastagens. De acordo com a Embrapa (2020), 80\% das pastagens brasileiras em níveis moderados e avançados enfrentam o problema da degradação. Como já mencionado a pressão por práticas sustentáveis, já não é mais tolerável que o extrativismo devaste áreas naturais e deixe-a inutilizada. 
Com o avanço das tecnologias, os mapeamentos por satélites ficarão cada vez mais precisos, aumentando o controle por partes reguladoras. Neste contexto, os pecuaristas se reinventam e adotam sistemas de integração como já mencionado, contando com incentivos governamentais para que até 2040 boa parte da área degrada já possa estar recuperada. Fatores tecnológicos são discussão para a produção através de ferramentas Smart Farming (SF) (PIVOTO et al., 2018); adoção de tecnologia para identificação de problemas como doenças (GROHER; HEITKÄMPER; UMSTÄTTER, 2020); e difusão de informações sobre a produção (CORTNER et al., 2019).

\subsection{Meio ambiente}

Aponta-se para o uso mais eficiente e aumento das fontes renováveis na agropecuária, incluindo bioenergia sustentável a partir de sistemas agroalimentares, isso fará com que o conceito de produzir sua própria energia se torne mais difundido no Brasil (EMBRAPA, 2020). Esta pratica pode ser a solução para deficiência no fornecimento de energia no campo, levada pelas redes convencionais das concessionárias, e seu alto custo (SILVA et al., 2017).

Outro ponto favorável a esta evolução é o retorno ao pecuarista e a contribuição para uma imagem da pecuária de forma mais sustentável e preocupada com o meio ambiente. É possível chegar a este objetivo pela intensificação da produção (CARDOSO et al., 2020); por modelos de bioeconomia (WETLESEN et al., 2020) e com a sua integração com outros sistemas produtivos (TAVANTI et al., 2020).

A adoção do sistema ILPF, melhor aporte nutricional dos bovinos e recuperação de áreas degradadas pela pecuária auxiliam na amenização da problemática dos impactos ambientais causados pela bovinocultura de corte (AVELINO et al., 2019). Outras soluções têm sido desenvolvidas como produtos de controle de fermentação ruminai, aditivos e probióticos para melhorar o manejo e a nutrição de bovinos, práticas como estas buscam reduzir o impacto ambiental da produção pecuária (MARTIN et al., 2016).

O Brasil pode aumentar seu consumo de água em $24 \%$ nos próximos dez anos de acordo com a agência do governo, em contrapartida especialistas da cadeia da carne acreditam que haja alta probabilidade de haver redução no consumo de água pela pecuária até 2040 (EMBRAPA, 2020). Adoção práticas de captação de água pluvial pelas propriedades rurais para reutilização em tratamento e possível consumo animal ou para limpeza de instalações e estudos acerca do 
melhor aproveitamento alimentar e hídrico, reduzindo desperdícios, são ações esperadas para os próximos anos (RODRIGUES; PRUSKI, 2019).

Um aumento de $6 \%$ de matas e florestas em relação à área total da agropecuária nos últimos 20 anos foram evidenciados no Censo Agropecuário do IBGE, havendo um tendência para a preocupação com questões ambientais (IBGE, 2017). A área de florestas cultivadas também aumentou 57,5\% nos últimos dez. A maior profissionalização da pecuária, a evolução do mercado madeireiro e de celulose, pressão de consumidores e racionalização do uso de terras são fatores que podem terminar o futuro da pecuária de corte (ALVES; MADARI; BODDEY, 2017).

\subsection{Comercialização de animais}

Em busca de maior lucratividade e melhores acordos comerciais, ou seja, maior competitividade na comercialização de animais, o setor de bovinocultura de corte no Brasil deve investir em especialização. Do ponto de vista produtivo e estrutural, para consolidar o agronegócio, deve-se apresentar uma concentração na produção, especialização e diversificação, resultando assim em um sistema agroindustrial em constante transformação.

Para tanto, faz-se necessário entender as particularidades de cada ciclo produtivo pecuário, pois cada um requer seu canal de compra e venda específico. Sendo estes bem variados, no qual a comercialização pode ocorrer desde a vizinhança com trocas de animais até os grandes contratos de boi gordo sendo negociado em plataformas digitais. Estima-se que até 2040 os dados de venda serão cada vez mais transparentes sobre os animais, os quais serão tratados por profissionais especializados em cada um dos segmentos: cria, recria e engorda (EMBRAPA, 2020).

Portanto, a fortificação das marcas das fazendas contribui para a maior profissionalização no comércio dos animais. A questão de boas práticas e bem-estar animal é uma temática que tem sido levantada pelo mercado externo e, é provável que até 2040 o pecuarista deva apresentar certificado, para possibilitar a comercialização (CEBALLOS et al., 2018). O bem-estar animal é averiguado por um conjunto de ações como a boa nutrição, boa saúde, bom manejo, instalações adequadas que geram bem-estar físico e mental do animal, ações estas que estão diretamente relacionadas a características que interessam ao setor de produção e comercialização (EMBRAPA, 2020).

Entretanto, a implementação e controle de tais práticas geram altos custos aos pecuaristas, para que estas mudanças ocorram em massa, um grande investimento proveniente 
do governo deverá ser feito para que o Brasil tenha atitudes mais rigorosas de regulamentação e melhorias no setor. Suporte e capital financeiro externo poderão ser diferenciais para a rentabilidade da produção (EROGLU; BOZOĞLU, 2019).

Altos investimentos também têm sido feitos na modernização dos leilões no país. Anteriormente o acesso aos animais era realizado unicamente presencialmente, após a criação de novas tecnologias existem então várias plataformas digitais de comunicação. Para maior rapidez de das negociações e variabilidade de acesso a animais, acredita-se que em no máximo dois anos a internet passará a televisão em comparação ao número de transmissão e acesso de leilões (EMBRAPA, 2020).

\subsection{Consolidação dos resultados}

Para a análise do conteúdo foi feita uma correlação da literatura levantada para analisar as tendencias para a produção de carne bovina. O quadro 2 apresenta a relação positiva (+) ou negativa (-) dos artigos analisados para com as tendencias identificadas pelo documento de visão para 2040.

A literatura abordada demonstra que as tendencias indicadas pelo estudo da Embrapa (2020) estão sendo discutidas globalmente, tendo uma relação positiva para a adesão dos novos modelos de produção de carne bovina, considerando juntamente, práticas cada vez mais sustentáveis que estão sendo cada vez mais demandadas pelas partes interessadas locais e globais.

As seis categorias discutidas a respeito do elo produtivo da cadeia da carne bovina tiveram relação positiva com as tendencias apresentadas pela Embrapa (2020). É importante destacar que os artigos discutidos, por sua vez, não trouxeram implicações empíricas de tendencias que abordassem todo o sistema da pecuária de corte, diferentemente como o documento aqui exposto. Destaca-se o estudo "Technical and economic efficiency of bovine weighing methods" (LOPES et al, 2018), que teve ralação negativa com a tendência "Orientação leva tecnologia ao campo" GT5, devido a fatores econômicos, onde o alto investimento em novas tecnologias não é bem aceito por uma parcela dos produtores pecuaristas, somados também a barreiras de fatores culturais do modo de produção. 
Quadro 2 - Resumo por categorias das literaturas correlacionadas com as tendências.

\begin{tabular}{|c|c|c|c|}
\hline Autores & Título & Tendência & $+/-$ \\
\hline \multicolumn{4}{|c|}{ Uso de Terras } \\
\hline $\begin{array}{l}\text { Vilpoux, } \\
\text { Gonzaga e } \\
\text { Pereira (2021) }\end{array}$ & $\begin{array}{l}\text { Agrarian reform in the Brazilian Midwest: Difficulties of modernization } \\
\text { via conventional or organic production systems }\end{array}$ & UT1, UT2 & + \\
\hline $\begin{array}{l}\text { Nunes et al. } \\
(2019)\end{array}$ & $\begin{array}{l}\text { Irreplaceable socioeconomic value of wild meat extraction to local food } \\
\text { security in rural Amazonia }\end{array}$ & UT1 & + \\
\hline $\begin{array}{l}\text { Stabile et al. } \\
(2020)\end{array}$ & $\begin{array}{l}\text { Solving Brazil's land use puzzle: Increasing production and slowing } \\
\text { Amazon deforestation }\end{array}$ & UT3 & + \\
\hline \multicolumn{4}{|c|}{ Gestão } \\
\hline \multirow{2}{*}{$\begin{array}{l}\text { Lopes et al. } \\
\text { (2018) }\end{array}$} & \multirow{2}{*}{ Technical and economic efficiency of bovine weighing methods } & GT1, G2, & + \\
\hline & & GT5 & - \\
\hline $\begin{array}{l}\text { Stabile et al. } \\
(2020)\end{array}$ & $\begin{array}{l}\text { Solving Brazil's land use puzzle: Increasing production and slowing } \\
\text { Amazon deforestation }\end{array}$ & GT2, GT5 & + \\
\hline $\begin{array}{l}\text { Barbosa et al. } \\
\text { (2020) }\end{array}$ & $\begin{array}{l}\text { Using Q-methodology to identify rural women's viewpoint on succession } \\
\text { of family farms }\end{array}$ & GT3 & + \\
\hline $\begin{array}{l}\text { Meneses e } \\
\text { Gomes (2020) }\end{array}$ & $\begin{array}{l}\text { Contemporary ruralities in the transamazônica region and xingu-pará } \\
\text { river: repercussions of schooling and environmentalization processes }\end{array}$ & GT3 & + \\
\hline $\begin{array}{l}\text { Ceballos et al. } \\
\text { (2018) }\end{array}$ & $\begin{array}{l}\text { Impact of good practices of handling training on beef cattle welfare and } \\
\text { stockpeople attitudes and behaviors }\end{array}$ & GT7 & + \\
\hline \multicolumn{4}{|c|}{ Estrutura produtiva } \\
\hline $\begin{array}{l}\text { Barbosa et al. } \\
\text { (2020) }\end{array}$ & $\begin{array}{l}\text { Using Q-methodology to identify rural women's viewpoint on succession } \\
\text { of family farms }\end{array}$ & EP1 & + \\
\hline $\begin{array}{l}\text { Oliveira et. Al } \\
\text { (2020) }\end{array}$ & $\begin{array}{l}\text { Greenhouse gas balance and carbon footprint of pasture-based beef } \\
\text { cattle production systems in the tropical region (Atlantic Forest biome) }\end{array}$ & EP4, EP5 & + \\
\hline \multicolumn{4}{|c|}{ Manejo } \\
\hline $\begin{array}{l}\text { Pivoto et al. } \\
\text { (2018) }\end{array}$ & $\begin{array}{l}\text { Scientific development of smart farming technologies and their } \\
\text { application in Brazil }\end{array}$ & MJ2 & + \\
\hline $\begin{array}{l}\text { Lampert et al. } \\
\text { (2019) }\end{array}$ & $\begin{array}{l}\text { Modelling beef cattle production systems from the Pampas in Brazil to } \\
\text { assess intensification options }\end{array}$ & $\begin{array}{l}\text { MJ1, MJ2, } \\
\text { MJ3, MJ6 }\end{array}$ & + \\
\hline $\begin{array}{l}\text { Aiken et al. } \\
(2020)\end{array}$ & $\begin{array}{l}\text { Forecasting beef production and quality using large-scale integrated data } \\
\text { from Brazil }\end{array}$ & MJ3 & + \\
\hline $\begin{array}{l}\text { Palhares et al } \\
(2021)\end{array}$ & $\begin{array}{l}\text { Water footprint of a tropical beef cattle production system: The impact } \\
\text { of individual-animal and feed management }\end{array}$ & MJ1, MJ3 & + \\
\hline $\begin{array}{l}\text { Ebling et al. } \\
\text { (2021) }\end{array}$ & $\begin{array}{l}\text { Event-based hydrology and sedimentation in paired watersheds under } \\
\text { commercial eucalyptus and grasslands in the Brazilian Pampa biome }\end{array}$ & MJ7 & + \\
\hline \multicolumn{4}{|c|}{ Meio ambiente } \\
\hline $\begin{array}{l}\text { Silva et al. } \\
\text { (2017) }\end{array}$ & $\begin{array}{l}\text { Analysis of the environmental perception and potential of the } \\
\text { photovoltaic panel in the perspective of poultry farmers of Toledo-PR }\end{array}$ & MA1 & + \\
\hline $\begin{array}{l}\text { Cardoso et al. } \\
(2020)\end{array}$ & $\begin{array}{l}\text { Intensification: A key strategy to achieve great animal and environmental } \\
\text { beef cattle production sustainability in Brachiaria grasslands }\end{array}$ & MA2 & + \\
\hline $\begin{array}{l}\text { Tavanti et al. } \\
\text { (2020) }\end{array}$ & $\begin{array}{l}\text { What is the impact of pasture reform on organic carbon compartments } \\
\text { and } \mathrm{CO} 2 \text { emissions in the Brazilian Cerrado? }\end{array}$ & MA2 & + \\
\hline $\begin{array}{l}\text { Avelino et al. } \\
\text { (2019) }\end{array}$ & $\begin{array}{l}\text { Phytonematodes in Integrated Crop-livestock Systems of Tropical } \\
\text { Regions }\end{array}$ & MA2, MA4 & + \\
\hline $\begin{array}{l}\text { Rodrigues e } \\
\text { Pruski (2019) }\end{array}$ & $\begin{array}{l}\text { Fundamentos e benefícios do sistema de integração lavoura-pecuária- } \\
\text { floresta para os recursos hídricos }\end{array}$ & MA3 & + \\
\hline $\begin{array}{l}\text { Alves, Madari e } \\
\text { Boddey (2017) }\end{array}$ & $\begin{array}{l}\text { Integrated crop-livestock-forestry systems: prospects for a sustainable } \\
\text { agricultural intensification }\end{array}$ & MA4 & + \\
\hline \multicolumn{4}{|c|}{ Comercialização de animais } \\
\hline $\begin{array}{l}\text { Ceballos et al. } \\
\text { (2018) }\end{array}$ & $\begin{array}{l}\text { Impact of good practices of handling training on beef cattle welfare and } \\
\text { stockpeople attitudes and behaviors }\end{array}$ & CA1, CA2 & + \\
\hline
\end{tabular}

Fonte: Dados da pesquisa.

Em se tratando da categoria "uso de terras" tem-se estudos confirmando as tendências apontadas, prezando pelo olhar e preocupações das nações, sob aspectos da expansão COLÓQUIO - Revista do Desenvolvimento Regional - Faccat - Taquara/RS - v. 19, n. 1, jan./mar. 2022 
agropecuária em detrimento da biodiversidade nativa. Quanto a "gestão", preza-se cada vez mais pela especialização dos atores envolvidos no setor produtivos da carne bovina, em busca de indicadores de desempenho atrelado aos incentivos econômicos, políticas públicas e práticas menos poluentes para manter a competitividade no mercado.

Ademais, a categoria "estrutura produtiva" confirma a tendência ILPF em expansão, assim como na "gestão", identificando a valorização futura do sistema de produção semi-intensivo e intensivo, os quais prezam pelo semiconfinamento e bem-estar animal. Por outro lado, também traz as tendencias alarmantes em relação ao êxodo rural. No tocante a categoria "manejo", confirma-se as tendências de disseminação cada vez mais ampla entre e para com os produtores a respeito de tecnificações e as demandas cada vez maiores de boas práticas.

No que concerne especificamente a categoria "meio ambiente" as tendencias reforçam o que outras categorias trazem como tendência decisões que atendam as questões de sustentabilidade ambiental, prezando por ferramentais que potencializem a produtividade e auxiliando na manutenção do meio ambiente. E, finalmente, a categoria "comercialização de animais" que sinalizam tendencias que corroboram com o bem-estar animal, tais como novas tecnologias sejam elas genéticas ou ferramentais.

Mesmo que a grande maioria das tendências tenham sido evidenciadas em pesquisas empíricas, alguns subtemas ainda são incipientes. Já outros não foram observados em pesquisas, como os subtemas acerca da gestão na ponta dos dedos; taurinos e zebuínos disputando a atenção do pecuarista; confinamento terceirizado facilitando a terminação de bovinos de corte; produtos de reuso são encontrados em instalações pecuárias; suplementação proteica via consócio ou via cocho; e a modernização dos leilões no Brasil. Estes subtemas ainda inexplorados devem se tornar temas emergentes para bovinocultura de corte brasileira para os próximos anos, bem como se tornar objeto de novas pesquisas.

\section{Considerações finais}

Objetivo deste artigo foi analisar o futuro da produção de bovinos de corte no Brasil em comparação com evidências já encontradas em bases internacionais. Foram buscados estudos evolvendo o contexto da bovinocultura de corte brasileira que se relacionavam com os cenários levantados pelo documento que analisou o futuro da cadeia produtiva da carne bovina brasileira para 2040. As implicações de um estudo amplo com o levantamento de tendências, são de relevância para que fornecedores de insumos, pecuaristas, frigoríficos comerciantes e 
consumidores estejam conscientes que mudanças necessárias estão acontecendo e que cada vez mais estarão inseridas no dia a dia do contexto da pecuária nacional.

No elo produção, o incremento de tecnologias é uma tendência em destaque, já que está evidente em todos os grandes temas de cenários futuros. Certamente há uma maior relação com gestão feita por pecuaristas mais jovens, treinados e orientados para o uso de tecnologias no campo, mas acaba se expandindo para o uso de terras, a estrutura produtiva, o manejo, o meio ambiente e a comercialização. Desse modo, a tecnologificação do campo se torna um elemento de ligação entre os cenários para a produção. Isso possibilita o aumento da produtividade e, ao mesmo tempo, a área de pastagem é reduzida e os impactos ao meio ambiente são mitigados.

Porém, a adoção de tecnologia ainda se mostra um obstáculo para grande parte dos pecuaristas, podendo se tornar um fator limitante para aplicação de fatores apontados nos cenários. Com custos elevados de implementação, necessidade de treinamento e manutenção, pequenos produtores e a agricultura familiar podem não conseguir acompanhar efetivamente as mudanças. Com isso, é importante a ação de associações de entidades de desenvolvimento governamentais por meio de políticas de incentivo aos pequenos produtores.

O documento base utilizado na análise desta pesquisa traz indícios de caminhos para pecuária para os próximos 20 anos. Estes são, além de tendências que irão acontecer de forma espontânea, possíveis eventos que necessitam de estabelecimento de estratégias e objetivos a serem alcançados. Portanto, pesquisas futuras devem acompanhar tais mudanças, desenvolvendo projetos e levando mais informação para o campo.

\section{Referências}

ABIEC. Perfil da pecuária no Brasil. BeefREPORT, p. 49, 2019.

AGROPENSA. Visão 2014-2034: 0 futuro do desenvolvimento tecnológico da agricultura brasileira. Brasília: Empresa Brasileira de Pesquisa Agropecuária Coordenação, 2014.

AIKEN, V. C. F. et al. Forecasting beef production and quality using large-scale integrated data from Brazil. Journal of Animal Science, v. 98, n. 4, abr. 2020.

ALVES, B. J. R.; MADARI, B. E.; BODDEY, R. M. Integrated crop-livestock-forestry systems: prospects for a sustainable agricultural intensification. Nutrient Cycling in Agroecosystems, v. 108, n. 1, p. 1-4, 2017.

AVELINO, A. C. D. et al. Phytonematodes in Integrated Crop-livestock Systems of Tropical Regions. Journal of Experimental Agriculture International, v. 37, n. 4, p. 1-13, 2019.

BARBOSA, R. A. et al. Using Q-methodology to identify rural women's viewpoint on succession of family farms. Land Use Policy, v. 92, n. January, p. 104489, 2020. 
BARDIN, L. Análise de Conteúdo. 1. ed. São Paulo: [s.n.].

CARDOSO, A. et al. Intensification: A key strategy to achieve great animal and environmental beef cattle production sustainability in Brachiaria grasslands. Sustainability (Switzerland), v. 12, n. 16, p. 1-17, 2020.

CEBALLOS, M. C. et al. Impact of good practices of handling training on beef cattle welfare and stockpeople attitudes and behaviors. Livestock Science, v. 216, p. 24-31, 2018.

CEPEA. O mercado de trabalho do agronegócio brasileiro, 2021.

CEZAR, I. M. et al. No Sistemas de Produção de Gado de Corte no Brasil: Uma Descricão com enfase no Regime Alimentar e no Abate. [s.l: s.n.]. v. 1

CEZIMBRA, I. M. et al. Potential of grazing management to improve beef cattle production and mitigate methane emissions in native grasslands of the Pampa biome. Science of The Total Environment, v. 780, p. 146582, ago. 2021.

CICARNE. Cadeia produtiva da carne bovina. Disponível em: <https://www.cicarne.com.br/2020/06/03/cadeia-produtiva-da-carne-bovina/>.

CORTNER, O. et al. Perceptions of integrated crop-livestock systems for sustainable intensification in the Brazilian Amazon. Land Use Policy, v. 82, n. December 2018, p. 841-853, 2019.

DÍAZ, S. et al. The IPBES Conceptual Framework - connecting nature and people. Current Opinion in Environmental Sustainability, v. 14, p. 1-16, jun. 2015.

DICK, M.; SILVA, M.; DEWES, H. Life cycle assessment of beef cattle production in two typical grassland systems of southern Brazil. Journal of Cleaner Production, v. 96, p. 426-434, 2015.

EBLING, É. D. et al. Event-based hydrology and sedimentation in paired watersheds under commercial eucalyptus and grasslands in the Brazilian Pampa biome. International Soil and Water Conservation Research, v. 9, n. 2, p. 180-194, jun. 2021.

EMBRAPA. O futuro da cadeia produtiva da carne bovina brasileira: uma visão para 2040. Disponível em: <https://www.embrapa.br/busca-de-publicacoes/-/publicacao/1125194/ofuturo-da-cadeia-produtiva-da-carne-bovina-brasileira-uma-visao-para-2040>.

EMBRAPA. Ciência que transforma. [s.l: s.n.].

ENAHORO, D. et al. Supporting sustainable expansion of livestock production in South Asia and Sub-Saharan Africa: Scenario analysis of investment options. Global Food Security, v. 20, p. 114-121, mar. 2019.

EROGLU, N. A.; BOZOĞLU, M. The effects of livestock supports and external input use on profitability of beef cattle farming: The case of samsun province, Turkey. Custos e Agronegocio, v. 15, n. 3, p. 368-383, 2019.

FAO. The future of food and agriculture: trends and challenges. Rome: Food and Agriculture Organization of the United Nations, 2017. v. 4

GROHER, T.; HEITKÄMPER, K.; UMSTÄTTER, C. Digital technology adoption in livestock production with a special focus on ruminant farming. Animal, v. 14, n. 11, p. 2404-2413, 2020.

$\mathrm{HU}, \mathrm{K}$. et al. Global research trends in food safety in agriculture and industry from 1991 to 2018: A data-driven analysis. Trends in Food Science and Technology, v. 85, n. December 2018, p. 262-276, 2019.

IBGE. Censo agropecuário. Disponível em: <https://censos.ibge.gov.br/agro/2017/>. 
IBGE. Censo agropecuário: resultados definitivos 2017 (IBGE, Ed.). Rio de Janeiro: [s.n.]. INSTITUTO BRASILEIRO DE GEOGRAFIA E ESTATÍSTICA, (IBGE). Censo Agropecuário 2017. Rio de Janeiro: [s.n.].

LAMPERT, V. DO N. et al. Modelling beef cattle production systems from the Pampas in Brazil to assess intensification options. Scientia Agricola, v. 77, n. 4, 2020.

LOPES, M. A. et al. Technical and economic efficiency of bovine weighing methods.

Semina:Ciencias Agrarias, v. 39, n. 3, p. 1167-1179, 2018.

MALAFAIA, G. C. et al. A sustentabilidade na cadeia produtiva da pecuária de corte brasileira. ILPF: inovação com integração de lavoura, pecuária e floresta, p. 117-130, 2019.

MARTIN, G. et al. Crop-livestock integration beyond the farm level: a review. Agronomy for Sustainable Development, v. 36, n. 3, 2016.

MDIC. Comextat - Carne bovina fresca, refrigerada ou congelada. Disponível em: $<$ http://comexstat.mdic.gov.br/>.

MENESES, A. S. DE; GOMES, R. A. CONTEMPORARY RURALITIES IN THE TRANSAMAZÔNICA REGION AND XINGU-PARÁ RIVER: REPERCUSSIONS OF SCHOOLING AND

ENVIRONMENTALIZATION PROCESSES. Revista Humanidades e Inovação, v. 7, n. 16, p. 184187, 2020.

NISKANEN, O.; IHO, A.; KALLIOVIRTA, L. Scenario for structural development of livestock production in the Baltic littoral countries. Agricultural Systems, v. 179, p. 102771, mar. 2020.

NUNES, A. V. et al. Irreplaceable socioeconomic value of wild meat extraction to local food security in rural Amazonia. Biological Conservation, v. 236, n. January, p. 171-179, 2019.

OLIVEIRA, P. P. A. et al. Greenhouse gas balance and carbon footprint of pasture-based beef cattle production systems in the tropical region (Atlantic Forest biome). Animal, v. 14, p. s427s437, 2020.

OTEROS-ROZAS, E. et al. Participatory scenario planning in place-based social-ecological research: insights and experiences from 23 case studies. Ecology and Society, v. 20, n. 4, p. art32, 2015.

OUKO, C. A. et al. Prospects of scenario planning for Kenya's protected ecosystems: An example of Mount Marsabit. Current Research in Environmental Sustainability, v. 1, p. 7-15, 2020.

PALHARES, J. C. P.; MORELLI, M.; NOVELLI, T. I. Water footprint of a tropical beef cattle production system: The impact of individual-animal and feed management. Advances in Water Resources, v. 149, p. 103853, mar. 2021.

PEARSON, T. R. H. et al. Greenhouse gas emissions from tropical forest degradation: an underestimated source. Carbon Balance and Management, v. 12, n. 1, p. 3, dez. 2017.

PIVOTO, D. et al. Scientific development of smart farming technologies and their application in Brazil. Information Processing in Agriculture, v. 5, n. 1, p. 21-32, 2018.

RODRIGUES, L. N.; PRUSKI, F. F. Fundamentos e benefícios do sistema de integração lavourapecuária-floresta para os recursos hídricos. Embrapa Cerrados-Capítulo em livro técnico (INFOTECA-E), 2019.

SILVA, A. A. L. DA et al. Analysis of the environmental perception and potential of the photovoltaic panel in the perspective of poultry farmers of Toledo-PR. Revista Produção e Desenvolvimento, v. 3, n. 1, p. 80-98, 2017. 
SOBROSA NETO, R. DE C. et al. An integrative approach for the water-energy-food nexus in beef cattle production: A simulation of the proposed model to Brazil. Journal of Cleaner Production, v. 204, p. 1108-1123, 2018.

STABILE, M. C. C. et al. Solving Brazil's land use puzzle: Increasing production and slowing Amazon deforestation. Land Use Policy, v. 91, n. May 2019, p. 104362, 2020.

TAVANTI, R. F. R. et al. What is the impact of pasture reform on organic carbon compartments and CO2 emissions in the Brazilian Cerrado? Catena, v. 194, n. May, p. 104702, 2020.

TERRITORIAL, E. Agricultura e preservação ambiental: uma análise do cadastro ambiental rural. Campinas - SP: [s.n.].

THEISSEN, T. et al. Environmental and socio-economic resources at the landscape level Potentials for sustainable land use in the Georgian Greater Caucasus. Journal of Environmental Management, v. 232, n. September 2018, p. 310-320, 2019.

USDA. United States Department of Agriculture - Market and trade data. Disponível em: $<$ https://www.fas.usda.gov/index.php>.

USDA. Livestock and poultry: world markets and trade. United States Department of Agriculture and Foreign Agricultural Service, p. 31, 2021b.

VILPOUX, O. F.; GONZAGA, J. F.; PEREIRA, M. W. G. Agrarian reform in the Brazilian Midwest: Difficulties of modernization via conventional or organic production systems. Land Use Policy, v. 103, n. February, p. 105327, 2021.

WETLESEN, M. S. et al. Simulations of feed intake, production output, and economic result within extensive and intensive suckler cow beef production systems. Livestock Science, v. 241, n. August, p. 104229, 2020.

$X U$, Z. et al. Energy modeling simulation of changes in ecosystem services before and after the implementation of a Grain-for-Green program on the Loess Plateau-A case study of the Zhifanggou valley in Ansai County, Shaanxi Province, China. Ecosystem Services, v. 31, p. 32-43, 2018.

ZU ERMGASSEN, E. K. H. J. et al. The origin, supply chain, and deforestation risk of Brazil's beef exports. Proceedings of the National Academy of Sciences of the United States of America, v. 117, n. 50, p. 31770-31779, 2020. 